\title{
Frequency Determination of Beams Coupled by a Double Spring-mass System Using Transfer Matrix Method of Linear Multibody Systems
}

\author{
Laith K. Abbas*, Qinbo Zhou, Xiaoting Rui \\ Institute of Launch Dynamics, Nanjing University of Science and Technology, Nanjing, 210094, China
}

\begin{abstract}
The analysis of natural vibration characteristics has become one of the important steps of the manufacture and dynamic design in different industries. The feasibility of using the transfer matrix method of linear multibody systems (MSTMM) to compute the free vibration characteristics of a system composed of continues and discrete elements vibrating in a plane is explored theoretically. A general analytical method based on MSTMM is developed for the frequencies determination of two Euler-Bernoulli beams coupled by a double spring-mass system. The components of the transfer matrix are all functions of the system's natural frequency. The overall transfer equation only involves boundary state vectors, whereas the state vectors at all other connection points do not appear. The state vectors at the boundary are composed of displacements, rotation angles, bending moments and shear forces, which are partly known and partly unknown. Recursive eigenvalue search algorithm is used to determine the system frequencies. A numerical example is presented to demonstrate the validity of the proposal method.
\end{abstract}

Keywords-coupled beams; transfer matrix method; spring-mass; recursive eigenvalue search; free vibration.

\section{INTRODUCTION}

There are large numbers of works in the literature on vibrations of combined dynamical systems consisting of beams to which spring-mass secondary systems are attached [1-2] using Green's functional method and other methods. The transfer matrix method of linear multibody systems (MSTMM) has been developed for a long time and has been used widely in structural analysis of linear and nonlinear systems. Applications of MSTMM range from vibration analysis, modeling of composite structures and multibody systems for computing static deformations, natural vibrations, steady state and transient dynamical response, to damage identification [3-7]. The general idea of MSTMM is to firstly break up a complicated system into elements with simple dynamic properties, which can be readily expressed in matrix form and treated in the same manner (for each element, it is possible to obtain the close form expression of the transfer matrix giving the displacements and the forces applied to one extremity to the displacements and forces applied to the other extremity). In other words, on an element level, the governing partial and ordinary differential or algebraic equations are transformed to algebraic transfer equations, where the output state results from a product of the input state and an element specific transfer matrix. These component matrices are considered as building blocks. In fact, the transfer matrix of such components does not need to be re-deduced, but may be taken directly from a transfer matrix library provided by [3]. These element transfer matrices are then assembled according to the topology of the flexible multibody system, ending up with a system of linear algebraic equations called the overall transfer equation. After eliminating the boundary conditions, the overall transfer matrix needs to be singular, which is checked by its determinant to be zero. Recursive eigenvalue search algorithm [7] is used to determine the system Eigen frequencies.

The present study is concerned essentially with the same mechanical system described in [2] but here the natural bending vibrations of two Bernoulli-Euler beams attached with a double spring-mass system are investigated using MSTMM.

\section{PROBLEM DESCRIPTION AND FORMULATION OF EIGENVALUE PROBLEM USING MSTMM}

The problem to be under study in this paper is the bending vibration problem of the system shown in Fig. 1. The system composed of continues elements and discrete element vibrating in a plane. It consists of two clamped-free Euler-Bernoulli beams carrying tip masses as the primary system to which a double spring-mass secondary system is attached across the span [2]. The length, mass per unit length, location of the spring attachment point, bending rigidity and tip mass of the $i$ th beam are $L_{i}, \bar{m}_{i}, \eta_{i} L_{i}, E_{i} I_{i}$, and $M_{i}$, respectively $(i=1,2)$. The secondary system consists of two lateral springs of stiffness $k_{1}, k_{2}$ and the mass $M$ [2]. The transfer equation between the input end and output end of any component in the system, for example, beam, spring, lumped mass can be stated as $\boldsymbol{Z}_{O}=\boldsymbol{U} \boldsymbol{Z}_{I}$, where $\boldsymbol{Z}$ is the state vector and $\boldsymbol{U}$ is the component transfer matrix. For the system vibrating in a plane, $\boldsymbol{Z}$ consists the state variables (SV) in the global coordinates including displacement $Y$, cross section small rotation angle $\Theta_{z}$, bending moment $M_{z}$ and shear force $Q_{y}$. The spring, lumped mass and beam transfer equations and matrices are respectively [3-7]: 


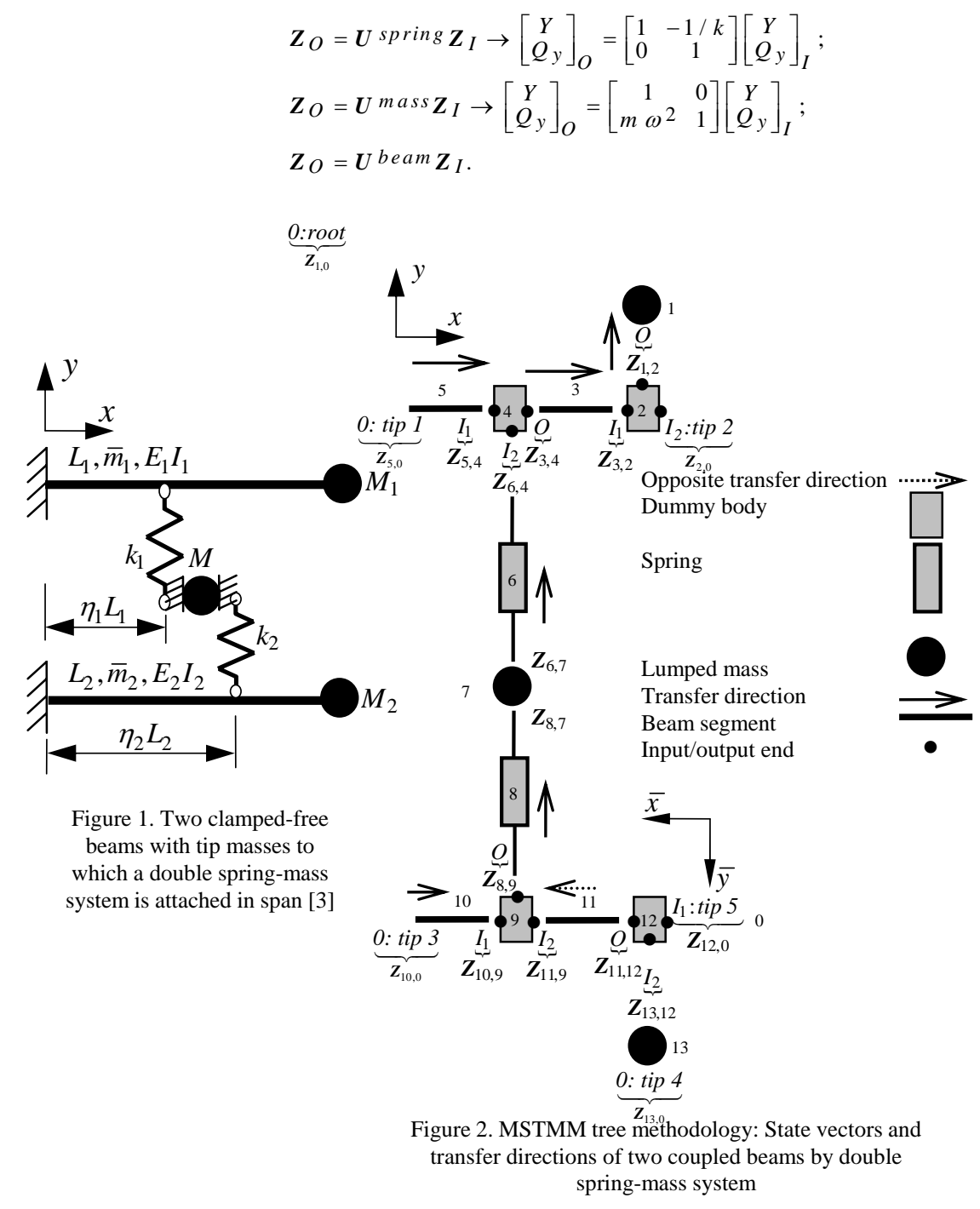

Herein, $m=\left\langle M\right.$ or $M_{1}$ or $\left.M_{2}\right\rangle$ and $\omega$ is the natural vibration frequency. A systematic MSTMM procedure is proposed which can be used to deal with the vibration problem of the structure shown in Fig.1. In MSTMM, there are different topologies of dynamics models such as chain, tree and closed-loop systems. In the context of MSTMM, the SVs and transfer directions of the complex structure shown in Fig. 1 are demonstrated in Fig. 2. For simplicity, the SV at the root is noted as $\boldsymbol{Z}_{1,0}$, while at the tip(s) is denoted as $\boldsymbol{Z}_{j, 0}$. Herein, subscript 0 notes the boundary and $j$ denotes the component boarders the boundary. The transfer directions of a system are always from the tip(s) to the root. The dotted arrows in Fig. 2 show the transfer directions for elements whose transfer direction do not match the orientation of the global reference frame $x y$. Thus, by introducing the local inertia reference frame $\overline{x y}$, the transfer equation for element stands in $\overline{x y}$. Therefore, these components need to transform from $\overline{x y} \rightarrow x y$ system coordinates. In Fig. 2, the vibrating system is a tree topology in the MSTMM algorithm and comprised of 13 components. Dummy rigid body (massless rigid body) components 2, 4, 9 and 12 are branches. It has 2 input ends and 1 output end. Components 3 , 5, 10 and 11 are beam segments. Spring components are 6 and 8. Lumped mass components are 1, 7 and 13. The entire system has totally 6 boundaries $\left(n_{b}=6\right)$, namely, $\boldsymbol{Z}_{1,0}, \boldsymbol{Z}_{5,0}, \boldsymbol{Z}_{2,0}, \boldsymbol{Z}_{10,0}, \boldsymbol{Z}_{13,0}$ and $\boldsymbol{Z}_{12,0}$ at the root, tips 1, 2, 3, 4 and 5, respectively. Following the MSTMM tree algorithm [9], the main transfer equation (TE) of the system is: 


$$
\begin{aligned}
& \boldsymbol{Z}_{1,0}=\boldsymbol{T}_{5-1} \boldsymbol{Z}_{5,0}+\boldsymbol{T}_{10-1} \boldsymbol{Z}_{10,0}+\boldsymbol{T}_{12-1} \boldsymbol{Z}_{12,0}+\boldsymbol{T}_{13-1} \boldsymbol{Z}_{13,0}+\boldsymbol{T}_{2-1} \boldsymbol{Z}_{2,0} \\
& \boldsymbol{T}_{5-1}=\boldsymbol{U}_{1} \boldsymbol{U}_{2, I_{1}} \boldsymbol{U}_{3} \boldsymbol{U}_{4, I_{1}} \boldsymbol{U}_{5} ; \quad \boldsymbol{T}_{10-1}=\boldsymbol{U}_{1} \boldsymbol{U}_{2, I_{1}} \boldsymbol{U}_{3} \boldsymbol{U}_{4, I_{2}} \boldsymbol{U}_{6} \boldsymbol{U}_{7} \boldsymbol{U}_{8} \boldsymbol{U}_{9, I_{1}} \boldsymbol{U}_{10} ; \\
& \boldsymbol{T}_{12-1}=\boldsymbol{U}_{1} \boldsymbol{U}_{2, I_{1}} \boldsymbol{U}_{3} \boldsymbol{U}_{4, I_{2}} \boldsymbol{U}_{6} \boldsymbol{U}_{7} \boldsymbol{U}_{8} \boldsymbol{U}_{9, I_{2}} \boldsymbol{C} \boldsymbol{U}_{11} \boldsymbol{C}^{-1} \boldsymbol{U}_{12, I_{1}} ; \\
& \boldsymbol{T}_{13-1}=\boldsymbol{U}_{1} \boldsymbol{U}_{2, I_{1}} \boldsymbol{U}_{3} \boldsymbol{U}_{4, I_{2}} \boldsymbol{U}_{6} \boldsymbol{U}_{7} \boldsymbol{U}_{8} \boldsymbol{U}_{9, I_{2}} \boldsymbol{C} \boldsymbol{U}_{11} \boldsymbol{C}^{-1} \boldsymbol{U}_{12, I_{2}} \boldsymbol{U}_{13} ; \quad \boldsymbol{T}_{2-1}=\boldsymbol{U}_{1} \boldsymbol{U}_{2, I_{2}}
\end{aligned}
$$

In (3), the subscript $j-1$ denotes the transfer direction from the component $j$ to the root component 1 . $\boldsymbol{U}_{3}, \boldsymbol{U}_{5}, \boldsymbol{U}_{10}$ and $\boldsymbol{U}_{11}$ are the transfer matrices of beam component $\boldsymbol{U}^{\text {Beam }}$. $\boldsymbol{U}_{6}$ and $\boldsymbol{U}_{8}$ are the transfer matrices of linear translational spring component $\boldsymbol{U}^{\text {spring }} . \boldsymbol{U}_{1}, \boldsymbol{U}_{7}$ and $\boldsymbol{U}_{13}$ are the transfer matrices of lumped mass component $\boldsymbol{U}^{\text {mass }} . \quad \boldsymbol{U}_{2, I_{1}}, \boldsymbol{U}_{2, I_{2}}, \boldsymbol{U}_{4, I_{1}}, \boldsymbol{U}_{4, I_{2}}, \boldsymbol{U}_{9, I_{1}}, \boldsymbol{U}_{9, I_{2}}, \boldsymbol{U}_{12, I_{1}}$ and $\boldsymbol{U}_{12, I_{2}}$ are the transfer matrices of massless rigid bodies 2, 4, 9 and 12, respectively, with multi-input and single output. The main transfer equation does not describe the geometrical relationship between different input ends and the number of unknown variables is more than that of algebraic equations. Therefore, another set of equations named geometrical equations (GEs) should be introduced, which describes the geometrical relationship between the first input and $(r=2,3,4, \ldots, L)$ input end of the component. GEs can be written in the following form $\boldsymbol{H}_{j, I_{1}} \boldsymbol{Z}_{j, I_{1}}=\boldsymbol{H}_{j, I_{r}} \boldsymbol{Z}_{j, I_{r}}$. The GEs corresponding to the dummy rigid bodies components 2 , 4, 9 and 12 can be deduced as follows, respectively:

$$
\begin{aligned}
& \boldsymbol{G}_{5-2} \boldsymbol{Z}_{5,0}+\boldsymbol{G}_{10-2} \boldsymbol{Z}_{10,0}+\boldsymbol{G}_{12-2} \boldsymbol{Z}_{12,0}+\boldsymbol{G}_{13-2} \boldsymbol{Z}_{13,0}+\boldsymbol{G}_{2-2} \boldsymbol{Z}_{2,0}=\mathbf{0} \\
& \boldsymbol{G}_{5-2}=-\boldsymbol{H}_{2, I_{1}} \boldsymbol{U}_{3} \boldsymbol{U}_{4, I_{1}} \boldsymbol{U}_{5} ; \quad \boldsymbol{G}_{10-2}=-\boldsymbol{H}_{2, I_{1}} \boldsymbol{U}_{3} \boldsymbol{U}_{4, I_{2}} \boldsymbol{U}_{6} \boldsymbol{U}_{7} \boldsymbol{U}_{8} \boldsymbol{U}_{9, I_{1}} \boldsymbol{U}_{10} ; \\
& \boldsymbol{G}_{12-2}=-\boldsymbol{H}_{2, I_{1}} \boldsymbol{U}_{3} \boldsymbol{U}_{4, I_{2}} \boldsymbol{U}_{6} \boldsymbol{U}_{7} \boldsymbol{U}_{8} \boldsymbol{U}_{9, I_{2}} \boldsymbol{C} \boldsymbol{U}_{11} \boldsymbol{C}^{-1} \boldsymbol{U}_{12, I_{1}} \text {; } \\
& \boldsymbol{G}_{13-2}=-\boldsymbol{H}_{2, I_{1}} \boldsymbol{U}_{3} \boldsymbol{U}_{4, I_{2}} \boldsymbol{U}_{6} \boldsymbol{U}_{7} \boldsymbol{U}_{8} \boldsymbol{U}_{9, I_{2}} \boldsymbol{C} \boldsymbol{U}_{11} \boldsymbol{C}^{-1} \boldsymbol{U}_{12, I_{2}} \boldsymbol{U}_{13} ; \quad \boldsymbol{G}_{2-2}=\boldsymbol{H}_{2, I_{2}} \\
& \boldsymbol{G}_{5-4} \boldsymbol{Z}_{5,0}+\boldsymbol{G}_{10-4} \boldsymbol{Z}_{10,0}+\boldsymbol{G}_{12-4} \boldsymbol{Z}_{12,0}+\boldsymbol{G}_{13-4} \boldsymbol{Z}_{13,0}=\mathbf{0} \\
& \boldsymbol{G}_{5-4}=-\boldsymbol{H}_{4, I_{1}} \boldsymbol{U}_{5} ; \quad \boldsymbol{G}_{10-4}=\boldsymbol{H}_{4, I_{2}} \boldsymbol{U}_{6} \boldsymbol{U}_{7} \boldsymbol{U}_{8} \boldsymbol{U}_{9, I_{1}} \boldsymbol{U}_{10} ; \\
& \boldsymbol{G}_{12-4}=\boldsymbol{H}_{4, I_{2}} \boldsymbol{U}_{6} \boldsymbol{U}_{7} \boldsymbol{U}_{8} \boldsymbol{U}_{9, I_{2}} \boldsymbol{C} \boldsymbol{U}_{11} \boldsymbol{C}^{-1} \boldsymbol{U}_{12, I_{1}} \text {; } \\
& \boldsymbol{G}_{13-4}=\boldsymbol{H}_{4, I_{2}} \boldsymbol{U}_{6} \boldsymbol{U}_{7} \boldsymbol{U}_{8} \boldsymbol{U}_{9, I_{2}} \boldsymbol{C} \boldsymbol{U}_{11} \boldsymbol{C}^{-1} \boldsymbol{U}_{12, I_{2}} \boldsymbol{U}_{13} \\
& \boldsymbol{G}_{10-9} \mathbf{Z}_{10,0}+\boldsymbol{G}_{12-9} \mathbf{Z}_{12,0}+\boldsymbol{G}_{13-9} \mathbf{Z}_{13,0}=0 \\
& \boldsymbol{G}_{10-9}=-\boldsymbol{H}_{9, I_{1}} U_{10} ; \quad G_{12-9}=H_{9, I_{2}} C U_{11} C^{-1} U_{12, I_{1}} ; \quad G_{13-9}=H_{9, I_{2}} C U_{11} C^{-1} U_{12, I_{2}} U_{13} \\
& \boldsymbol{G}_{12-12} \boldsymbol{Z}_{12,0}+\boldsymbol{G}_{13-12} \boldsymbol{Z}_{13,0}=\mathbf{0} \\
& \boldsymbol{G}_{12-12}=-\boldsymbol{H}_{12, I_{1}} ; \quad \boldsymbol{G}_{13-12}=\boldsymbol{H}_{12, I_{2}} \boldsymbol{U}_{13} .
\end{aligned}
$$

The equilibrium condition of the moments acted on the dummy bodies 2 and 9 are still missing, that is: $\boldsymbol{m}_{2, I_{1}}+\boldsymbol{m}_{2, I_{2}}=\mathbf{0}$ and $\boldsymbol{m}_{9, I_{1}}+\boldsymbol{m}_{9, I_{2}}=\mathbf{0}$, respectively.

Consequently, the so-called supplementary equations (SEs) can be obtained for bodies 2 and 9, respectively:

$$
\begin{aligned}
& S_{5-2} Z_{5,0}+S_{10-2} Z_{10,0}+S_{12-2} Z_{12,0}+S_{13-2} Z_{13,0}+S_{2-2} Z_{2,0}=0 \\
& S_{5-2}=-E_{2, I_{1}} U_{3} U_{4, I_{1}} U_{5} ; \quad S_{10-2}=-E_{2, I_{1}} U_{3} U_{4, I_{2}} U_{6} U_{7} U_{8} U_{9, I_{1}} U_{10} ; \\
& S_{12-2}=-E_{2, I_{1}} U_{3} U_{4, I_{2}} U_{6} U_{7} U_{8} U_{9, I_{2}} C U_{11} C^{-1} U_{12, I_{1}} ; \\
& S_{13-2}=-E_{2, I_{1}} U_{3} U_{4, I_{2}} U_{6} U_{7} U_{8} U_{9, I_{2}} C U_{11} C^{-1} U_{12, I_{1}} U_{13} ; \quad S_{2-2}=E_{2, I_{2}} \\
& S_{10-9} Z_{10,0}+S_{12-9} Z_{12,0}+S_{13-9} Z_{13,0}=0 \\
& S_{10-9}=-E_{9, I_{1}} U_{10} ; \quad S_{12-9}=E_{9, I_{2}} C U_{11} C^{-1} U_{12, I_{1}} ; \quad S_{13-9}=E_{9, I_{2}} C U_{11} C^{-1} U_{12, I_{2}} U_{13} .
\end{aligned}
$$

To the end, by combining (3, 4 and 5), the overall transfer equation of the entire system shown in Fig. 2 can be obtained:

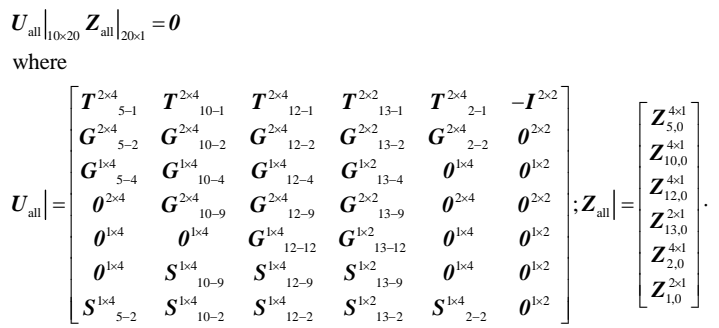

At the boundaries, half of these SVs are zero due to known constraints

which are $\quad Z_{1,0}=\left[\begin{array}{ll}Y & 0\end{array}\right]^{T} \quad, \quad Z_{2,0}=\left[\begin{array}{llll}Y & \Theta_{Z} & 0 & 0\end{array}\right]^{T}$, $\boldsymbol{Z}_{5,0}=\left[\begin{array}{llll}0 & 0 & M_{z} & Q_{y}\end{array}\right]^{T}, \quad Z_{10,0}=\left[\begin{array}{llll}0 & 0 & M_{z} & Q_{y}\end{array}\right]^{T}$, $Z_{12,0}=\left[\begin{array}{llll}Y & \Theta_{z} & 0 & 0\end{array}\right]^{T}$ and $Z_{13,0}=\left[\begin{array}{ll}Y & 0\end{array}\right]^{T}$. Thus, (6) reduces to $\overline{\boldsymbol{U}}_{\text {all }} \overline{\boldsymbol{Z}}_{\text {all }}=\mathbf{0}$ where $\overline{\boldsymbol{Z}}_{\text {all }}$ is composed of the unknown elements only and $\overline{\boldsymbol{U}}_{\text {all }}$ is a square matrix resulting from elimination of all columns of $\boldsymbol{U}_{\text {all }}$ associated with zeros in $\boldsymbol{Z}_{\text {all }}$. Finally, $\overline{\boldsymbol{U}}_{\text {all }}$ is only a highly nonlinear algebraic function of the unknown natural frequency. For non-trivial solutions, the characteristic equation

$\Delta(\omega)=\operatorname{det} \overline{\boldsymbol{U}}_{\text {all }} \stackrel{!}{=} 0$ has to be fulfilled. Recursive eigenvalue search algorithm [7] is employed to determine the system frequencies.

\section{NUMERICAL EXAMPLE AND DISCUSSION}

The reliability of the theoretical work using MSTMM with tree scenario and recursive scanning algorithm in the context of free vibration is validated with the example in [3] as shown in Fig. 1. The elastically coupled beams have the following geometrical and material properties: $L_{1}=L_{2}=1 \mathrm{~m}$, $E_{1} I_{1}=E_{2} I_{2}=166.67 \mathrm{Nm}^{2}$ and $\bar{m}_{1}=\bar{m}_{2}=0.78 \mathrm{~kg} / \mathrm{m}$. The positions of the two springs, linear translational stiffness of the two springs, and lumped masses values have been considered similar to [3] as follows: $\eta_{1}=\eta_{2}=0.5, k_{1}=1000 \times E_{1} I_{1} / L_{1}^{3}, k_{2}=1000 \times E_{2} I_{2} / L_{2}^{3}$, $M=1 \times \bar{m}_{1} \times L_{1} \quad, M_{1}=2 \times \bar{m}_{1} \times L_{1}$ and $M_{2}=2 \times \bar{m}_{2} \times L_{2}$, respectively. Applying fMin1D algorithm $\left([1,3000], N_{x 0}=2000, \varepsilon=10^{-8}\right) \quad$ [7], the absolute value of the determinant is shown in Fig. 3. The first ten non-dimensionless frequency values $\left(\lambda_{i}=\sqrt[4]{\bar{m}_{1} \omega_{i}^{2} / E_{1} I_{1}}\right)$ of the entire system are presented in Table I. Summarizing, MSTMM results are in good agreement with [2] and it's able to handle this kind of problems. 
TABLE I. THE FIRST TEN NON-DIMENSIONAL FREQUENCY PARAMETERS $\lambda$ OF THE SYSTEM SHOWN IN FIG. 1 IN COMPARISON WITH [2]

\begin{tabular}{|c|c|c|}
\hline & MSTMM & {$[3]$} \\
\hline 1 & 1.070262867381579 & 1.07026286 \\
\hline 2 & 1.558789833913990 & 1.55878983 \\
\hline 3 & 3.309591525266765 & 3.30959152 \\
\hline 4 & 6.334254798565825 & 6.33425479 \\
\hline 5 & 6.819830366275476 & 6.81983036 \\
\hline 6 & 7.469383234453217 & 7.46938324 \\
\hline 7 & 7.925955951118066 & 7.92595594 \\
\hline 8 & 10.666735836264996 & 10.66673582 \\
\hline 9 & 10.746971283874736 & 10.74697127 \\
\hline 10 & 13.402318464745367 & 13.40231846 \\
& & \\
\hline
\end{tabular}

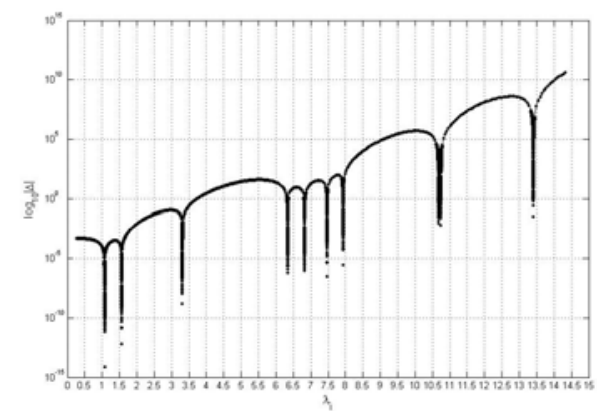

Figure 3. Root scanning of a specific frequency band for a system in Fig. 2

\section{CONCLUSIONS}

This study deals with the bending free vibrations of a system where two beams are coupled by a double spring-mass system. To determine the system Eigen characteristics, the transfer matrix method of linear multibody systems has been proposed in this paper. It should be noted that there is no inherent difficulty in extending the current method to a system consisting of any number of uniform or non-uniform beams, springs and dampers. Since the solution is almost exact and the results are interesting, this allows a complete understanding of the problem. The proposal method is easy to formulate, systematic to apply and simple to code.

\section{ACKNOWLEDGMENT}

The research was supported by the Research Fund for the Doctoral Program of Higher Education of China (20113219110025, 20133219110037), the Natural Science Foundation of China Government (11102089, 61304137), and the Program for New Century Excellent Talents in University (NCET-10-0075).

\section{REFERENCES}

[1] Mermertas, V. Alternative formulations of the frequency equation of longitudinally vibrating rods coupled by a double spring-mass system, Journal of Sound and Vibration, 208, pp. 331-338, 1997.

[2] Gurgoze, M., Erdogan, G. \& Inceoglu, S. Bending vibrations of beams coupled by a double spring-mass system, Journal of Sound and Vibration, 243, pp. 361-369, 2001.

[3] Rui, X., Yun, L., Lu, Y., He, B. \& Wang, G. Transfer Matrix Method of Multibody System and its Application. Beijing: Beijing Science Press, 2008 (in Chinese).

[4] Abbas, L. K., Li, J. \& Rui, X. Transfer matrix method for the determination of the natural vibration characteristics of realistic thrusting launch vehicle-Part I, Mathematical Problems in Engineering, 2013, Article ID 764673, 16 pages, 2013.

[5] Abbas, L. K., Bestle, D. \& Rui, X. Transfer matrix method for the determination of the free vibration of two elastically coupled beams, Applied Mechanics and Materials, 372, pp. 301-304, 2013.

[6] Abbas, L. K. \& Rui, X. Free vibration characteristic of multi-level beam based on transfer matrix method of multibody systems, Advances in Mechanical Engineering-Special Issue "New developments in multibody system dynamics and its applications", 2014, Article ID 792478, 16 pages, 2014.

[7] Bestle, D., Abbas, L. K. \& Rui, X. Recursive eigenvalue search algorithm for transfer matrix method of flexible multibody systems, Multibody System Dynamics, 32 , pp. 429-444, 2014. 